\title{
Effect of the sample geometry on the second magnetization peak in single crystalline $\mathrm{Ba}_{0.63} \mathrm{~K}_{0.37} \mathrm{BiO}_{3}$ thick film
}

\author{
A. Yu. Galkin ${ }^{\mathrm{a}, 1}$, Y. Kopelevich ${ }^{\mathrm{a}, 2}$, P. Esquinazi ${ }^{\mathrm{a}}$ A. Setzer ${ }^{\mathrm{a}}$ \\ V. M. Pan ${ }^{\mathrm{b}}$ and S. N. Barilo ${ }^{\mathrm{c}}$

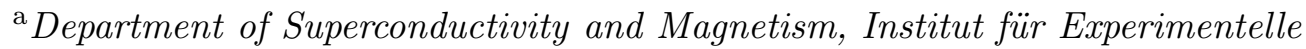 \\ Physik II, Universität Leipzig, Linnéstr. 5, D-04103 Leipzig, Germany \\ ${ }^{\mathrm{b}}$ Institute of Metal Physics, National Academy of Sciences, Vernadsky str.36, Kiev \\ 252041, Ukraine \\ ${ }^{\mathrm{c}}$ Institute of Physics of Solids and Semiconductors, Academy of Science, Minsk \\ 220072, Belarus
}

\begin{abstract}
Magnetization hysteresis loop $M(H)$ measurements performed on a single crystalline $\mathrm{Ba}_{0.63} \mathrm{~K}_{0.37} \mathrm{BiO}_{3}$ superconducting thick film reveal pronounced sample geometry dependence of the "second magnetization peak" (SMP), i.e. a maximum in the width of $M(H)$ occurring at the field $H_{\mathrm{SMP}}(T)$. In particular, it is found that the SMP vanishes decreasing the film dimension. We argue that the observed sample geometry dependence of the SMP cannot be accounted for by models which assume a vortex pinning enhancement as the origin of the SMP. Our results can be understood considering the thermomagnetic instability effect and/or non-uniform current distribution at $H<H_{\mathrm{SMP}}$ in large enough samples.
\end{abstract}

Recently, the dependence of the second magnetization peak (SMP) - a maximum in the width of the magnetization hysteresis loop - on the sample geometry has been reported for $\mathrm{Nb}$ films $[1,2]$ and $\mathrm{Bi}_{2} \mathrm{Sr}_{2} \mathrm{CaCu}_{2} \mathrm{O}_{8}$ single crystals [3]. In particular, it was found that the SMP vanishes in both superconductors for small enough dimensions of the samples. The occurrence of the SMP in

1 On leave from Institute of Metal Physics, National Academy of Sciences, Kiev 252041, Vernadsky str.36, Ukraine. Supported by the Sächsiches Staatsministerium für Wissenschaft und Kunst

2 On leave from Instituto de Fisica, Unicamp, 13083-970 Campinas, Sao Paulo, Brasil. Supported by the Deutsche Forschungsgemeinschaft under DFG IK 24/B11, Project H, and by CAPES proc. No. 077/99.

Preprint submitted to Elsevier Preprint 16 October 2018 


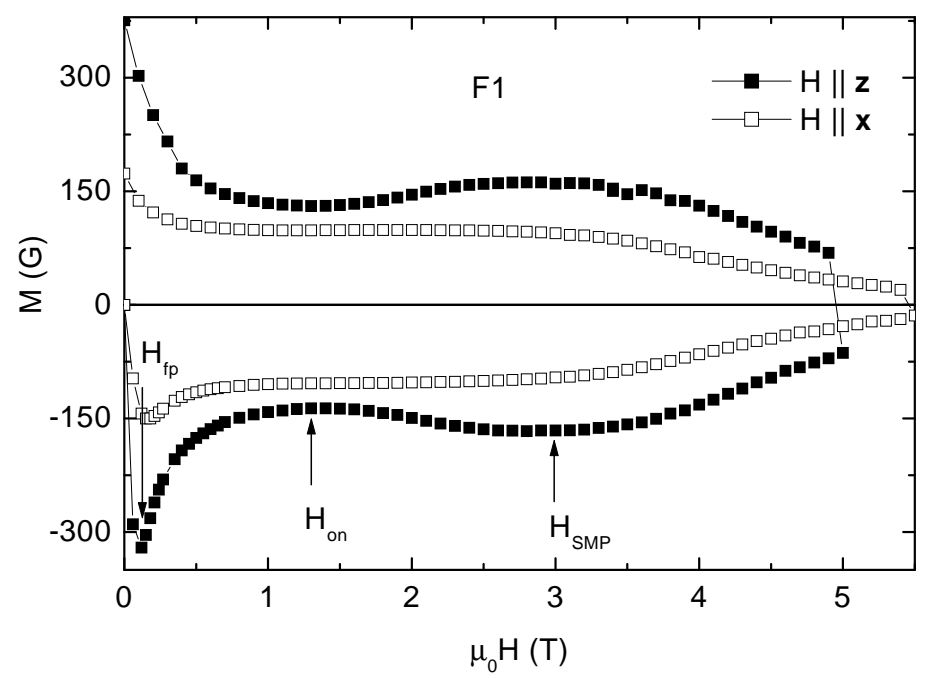

Fig. 1. Positive field region of magnetization hysteresis loops measured in the virgin film (sample F1) perpendicular $(H \| z)$ and parallel $(H \| x)$ to the main surface of the film. For $H \| z$ configuration we indicate the magnetic fields corresponding to the first $H_{\mathrm{fp}}$ and second $H_{\mathrm{SMP}}$ magnetization peaks, as well as the SMP onset field $H_{\text {on }}$.

large samples has been interpreted in terms of the thermomagnetic instability (TMI) effect that leads to a reduced, as compared to the isothermal critical state value, irreversible magnetization at $H<H_{\mathrm{SMP}}(T)$, where $H_{\mathrm{SMP}}(T)$ corresponds to the field at the SMP. On the other hand, Gurevich and Vinokur (GV) [4] have suggested that the SMP can result from a non-uniform current flow at $H<H_{\mathrm{SMP}}(T)$ due to material inhomogeneities, without invoking TMI effects. This model [4] also predicts the vanishing of the SMP decreasing the sample size. Thus, the interpretations of SMP given in Refs. [1-4] are fundamentally different from those based on an increase of the critical current density, implying that a careful distinction between the different approaches is necessary. The study of the sample geometry dependence of SMP is one of the experimental instruments allowing for such a distinction.

The purpose of the present work is to verify the effect of the sample geometry on the SMP in the non-cuprate, isotropic high- $T_{c}\left(T_{c} \simeq 30 \mathrm{~K}\right)$ superconductor $(\mathrm{Ba}, \mathrm{K}) \mathrm{BiO}_{3}$. The here studied sample is a $\mathrm{Ba}_{0.63} \mathrm{~K}_{0.37} \mathrm{BiO}_{3}$ single crystalline thick film grown on $\mathrm{BaBiO}_{3}$ single crystal as a seed. The details of the film preparation are described elsewhere [5]. The good quality of the film was confirmed by x-ray diffraction measurements (rocking curve FWHM $\leq 0.4^{\circ}$ ). The virgin film with dimensions $a \times b \times c=2.9 \times 2.2 \times 0.25 \mathrm{~mm}^{3}$, labeled as F1, was structured with the help of a thin wire in 10 stripes of size $0.12 \times 2.2 \times$ $0.25 \mathrm{~mm}^{3}$, i.e. with the cuts parallel to the sample b- direction. This sample is labeled as F2. Then, the stripes size was further reduced to $120 \times 120 \times 250 \mu \mathrm{m}^{3}$. 


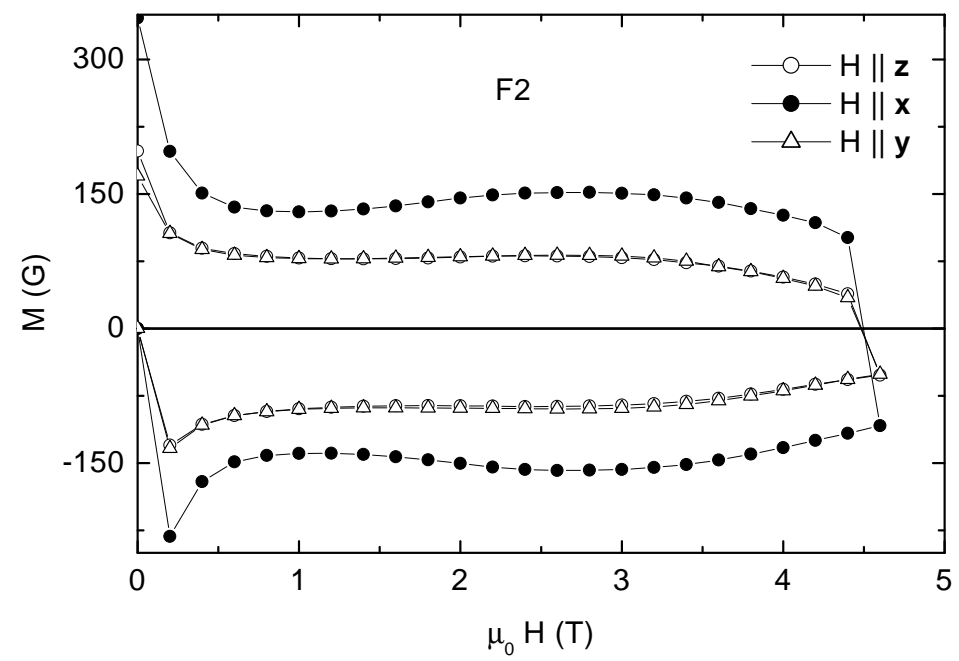

Fig. 2. Positive field region of magnetization hysteresis loops measured in the sample F2 for the three orthogonal field directions.

This sample, consisting of small-size bars, is labeled as F3.

Isothermal magnetic hysteresis loop $m(H)$ measurements $(m(H)$ is the sample magnetic moment) were performed using a SQUID magnetometer (Quantum Design-MPMS7). The measurements were always made after cooling the sample in a zero applied magnetic field to the target temperature. In this work we restrict the presentation and discussion of the results obtained at $T=5 \mathrm{~K}$.

Figure 1 shows magnetization curves $M(H)=m(H) / V$ ( $V$ is the sample volume) measured with $H \| x$ and $H \| z$ (here and below $x, y$ and $z$ axes are directed along $a, b$ and $c$ dimensions of the virgin film, respectively). As seen in Fig. 1, the virgin magnetization $|M(H)|$ shows a local maximum at $\mu_{0} H_{\mathrm{fp}} \simeq$ $0.1 \mathrm{~T}$, decreases with field and reaches a minimum at $\mu_{0} H_{\mathrm{on}} \simeq 1.3 \mathrm{~T}$ and peaks again at $\mu_{0} H_{\mathrm{SMP}} \simeq 3 \mathrm{~T}$ (the SMP onset field $H_{\mathrm{on}}$ is nearly temperature independent at $T<20 \mathrm{~K}$ ).

The first hint on the sample geometry dependence of the SMP comes from the pronounced difference in the hysteresis loops measured in the sample F1 with the field oriented perpendicular and parallel to the film's main surface. Indeed, as Fig. 1 demonstrates, the SMP is pronounced for the transverse $(H \| z)$ geometry, whereas it is small for the parallel field configuration $(H \| x)$. It has been shown, however, that the seeded growth- induced anisotropy may affect the SMP [6]. To check this we have measured $M(H)$ in the sample F2 for all three field orientations $(H\|x, H\| y$, and $H \| z)$. Results of these measurements, given in Fig. 2, indicate that a well defined SMP takes place for $H \| x$, i. e. again for the transverse geometry, whereas for $H \| y$ and $H \| z$, 


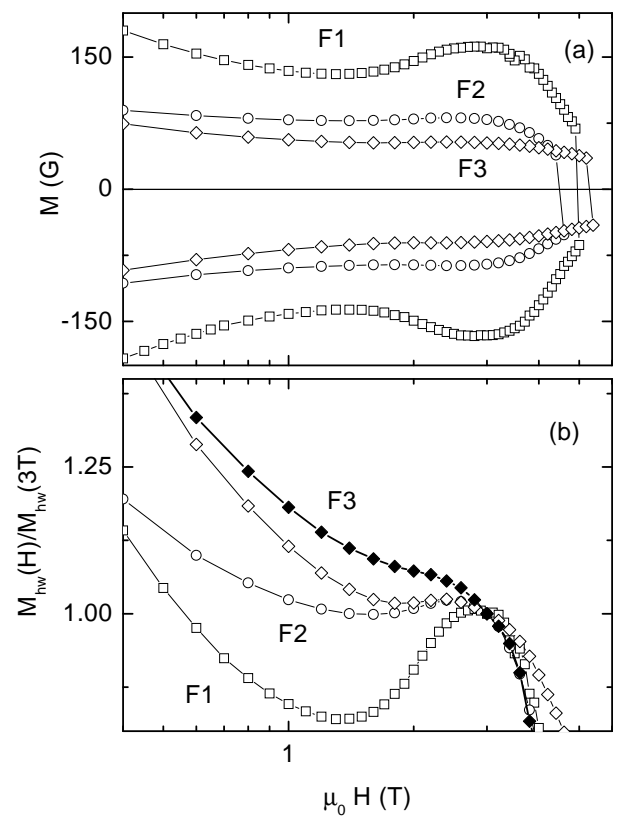

Fig. 3. (a) Positive field region of magnetization hysteresis loops measured in the samples F1, F2, and F3 with $H \| z$, and plotted in a semilogarithmic scale. (b) Half-width of the hysteresis loops $M_{\mathrm{hw}}(H)$ obtained for the samples F1, F2, F3 and normalized by the value at $\mu_{0} H=3 \mathrm{~T}$. $H \| z$ : open symbols, $H \| x$ : solid symbols (only for the sample F3).

the SMP is rather weak. This implies that the SMP is determined by the sample geometry, while the possible growth-induced anisotropy is irrelevant.

Figure 3(a) shows $M(H)$ measured in the samples F1, F2, and F3 with $H \| z$, i.e. with the field direction parallel to the unchanged sample dimension $(c=250 \mu \mathrm{m})$. Figure 3(b) shows the half-width of the hysteresis loops $M_{\mathrm{hw}}(H)=\left[M^{+}(H)-M^{-}(H)\right] / 2$ obtained for the three samples and normalised by its value at $\mu_{0} H=3 \mathrm{~T}\left(M^{+}(H)\right.$ and $M^{-}(H)$ are the magnetisation corresponding to the ascending and descending branches of the hysteresis loop). For completeness, $M_{\mathrm{hw}}(H) / M_{\mathrm{hw}}(3 T)$ obtained for the sample F3 with $H \| x$ is also given in Fig. 3(b). As Fig. 3(a,b) demonstrates, the SMP is almost completely suppressed in the sample F3 which consists of the smallest-size bars.

We stress, that the hysteresis loops measured in all samples and for different field orientations are symmetrical with respect to the zero magnetization axis. This indicates that the hysteretic mechanism is dominated by bulk vortex properties as well as that the pinning induced irreversible magnetization $\left|M_{\text {irr }}(H)\right|$ is much larger than the equilibrium magnetization $\left|M_{\text {eq }}(H)\right|$. Thus, $\left|M_{\mathrm{hw}}(H)\right|\left(\sim\left|M_{\mathrm{irr}}(H)\right|\right)$ is proportional to the critical current density $j_{c}(H)$ 
which can be calculated using the Bean model for either transverse or longitudinal geometry [7]. We estimate for our samples $j_{c} \sim 10^{9} \mathrm{~A} / \mathrm{m}^{2}$ at $T=5 \mathrm{~K}$, which slightly varies in the field interval $H_{\mathrm{fp}}<H<H_{\mathrm{SMP}}$.

The magnetization time relaxation $M_{\mathrm{irr}}(t)$ measured in this work demonstrates that the vortex creep at $5 \mathrm{~K}$ only weakly affects $j_{c}$. The obtained logarithmic relaxation rate at $H<H_{\mathrm{SMP}}, \beta=-\mathrm{d}\left(\log \left(M_{\mathrm{irr}}\right)\right) / \mathrm{d}(\log (t)) \sim 0.02$ is in agreement with previous reports [8].

We start the discussion of the experimental results comparing the sample dimensions to the relevant vortex-pining-related characteristic lengths. At $H \sim H_{\text {on }} \sim 1 \mathrm{~T}\left(<<H_{c 2}(0)\right)[6]$ and taking the London penetration depth $\lambda(0) \sim 0.3 \mu \mathrm{m}$, coherence length $\xi(0) \sim 4 \mathrm{~nm}$, the Ginzburg-Landau parameter $\kappa=\lambda / \xi \sim 75[6]$, and $j_{c} \sim 10^{9} \mathrm{~A} / \mathrm{m}^{2}$, we obtain:

- the longitudinal collective pinning length [7] $L_{c}=\left(c_{44} / c_{66}\right)^{1 / 2} R_{c} \sim 3 \mu \mathrm{m}$,

- the transverse correlation radius $R_{c}=8 \pi r_{p}^{2} c_{44}^{1 / 2} c_{66}^{3 / 2} / W \sim 50 \mathrm{~nm}$ (with the range of the pinning force $r_{p} \simeq \xi$ ),

- the longitudinal size of the elastic cage $L_{0}=\left(\epsilon_{0} / c_{66}\right)^{1 / 2} \sim 0.2 \mu \mathrm{m}$, appearing in the theory of the disorder-driven transition between the flux-line-lattice (FLL) and the entangled vortex state $[9,10]$.

Here $c_{44} \simeq H^{2} \mu_{0}$ and $c_{66} \simeq \Phi_{0} H / 16 \pi \lambda^{2}$ are the FLL tilt and shear moduli, respectively, $\epsilon_{0}=\left(\Phi_{0}^{2} / 4 \pi \mu_{0} \lambda^{2}\right) \ln (\kappa)$ is the vortex line energy, $W=$ $\left(F_{p} r_{p}^{3} c_{66}^{2} c_{44}\right)^{1 / 2} /\left(1.5^{1 / 2} / 32 \pi^{2}\right)^{1 / 2}$ the parameter which measures the pinning strength [7], and $F_{p}=B j_{c}$ the pinning force. Hence we conclude that the reduction of the sample dimension to $\sim 100 \mu \mathrm{m}$ cannot affect the pinning efficiency of vortices. Thus, the observed sample size dependence of the SMP is clearly against an interpretation of the SMP based on an increase of $j_{c}(H)$. Note also that $L_{c} \gg L_{0}$, i.e. vortices are in a different pinning regime from that discussed in Refs. $[9,10]$.

Alternatively, Refs. [1-4] treat the SMP as resulting from either a TMI effect [1-3] or from a non-uniform current flow due to material inhomogeneities [4] which takes place at $H<H_{\mathrm{SMP}}$. According to the TMI theory, the condition for thermomagnetic stability is achieved for an effective sample dimension $s_{\text {eff }}<s_{\text {crit }}=10 H_{\mathrm{fj}} / 4 \pi j_{c}[11]$, where $H_{\mathrm{fj}}$ is the field at which the first magnetization jump (or reduction of the isothermal critical state magnetization which can occur in a rather smooth fashion even in Nb films [1]) takes place. Within our interpretation (see also Refs. [1-3]), $H_{\mathrm{fj}} \simeq H_{\mathrm{fp}}$. Thus, taking the experimental values for $\mu_{0} H_{\mathrm{fp}} \sim 0.1 \mathrm{~T}$ and $j_{c} \sim 10^{9} \mathrm{~A} / \mathrm{m}^{2}$, we get $s_{\text {crit }} \sim 100 \mu \mathrm{m}$. For the sample $\mathrm{F} 1$ in the transverse geometry, $H \| z, s_{\text {eff }} \sim(b c / 2)^{1 / 2} \sim 500 \mu \mathrm{m}$ [12], i.e. $s_{\text {eff }} \gg s_{\text {crit }}$. Therefore, a pronounced SMP is expected as observed in this geometry (see Fig. 1).

Similarly, for $H \| x$ the effective sample (F1) size [11] $s_{\mathrm{eff}}=c / 2=125 \mu \mathrm{m}$, 
i.e. $s_{\text {eff }} \sim s_{\text {crit }}$. In this case, we would expect an essential suppression of the SMP as compared to the transverse geometry, which is also in agreement with the experimental observation (see Fig. 1). For an intermediate geometry $s_{\text {eff }}$ cannot be easily estimated. For instance, the case of the sample F2 (long stripes with a nearly square cross section) with $H \| x$, resembles that of a wire in a field applied normal to the wire axis, where the development of the SMPlike feature can be recognized as the length of the wire increases (see Fig. 3 in Ref. [13]). Figure $3(\mathrm{a}, \mathrm{b})$ clearly demonstrates that the SMP ultimately vanishes as the sample dimensions become of the order of $\sim 100 \mu \mathrm{m}$.

On the other hand, in Ref. [4] the hollow in the magnetization hysteresis loop in the field interval $H_{\mathrm{fp}}<H<H_{\mathrm{SMP}}$, has been attributed to a non-uniform current flow due to material inhomogeneties with a spatial scale $L \gg L_{c}$. This model predicts the vanishing of the SMP when the sample width becomes smaller than $w_{c} \sim L \sqrt{n}$, where $n=(1+\beta) / \beta$. Taking $w_{c} \sim 100 \mu \mathrm{m}$ and the experimental value for $n \sim 50(\beta \sim 0.02)$ we get $L \sim 14 \mu \mathrm{m}$. Then the question arises: is the size of the inhomogenities specific to our sample or it has a more general origin?

It is interesting to note that the SMP was essentially suppressed reducing the lateral sample size to $\sim 100 \mu \mathrm{m}$ also in $\mathrm{Nb}$ films [2] and $\mathrm{Bi}_{2} \mathrm{Sr}_{2} \mathrm{CaCu}_{2} \mathrm{O}_{8}$ single crystals [3], suggesting (within the framework of the GV theory) a similar scale length $L$ in these otherwise different superconductors. It is tempting to associate this fact with the irregular fractal-type flux penetration observed at low fields in various superconductors such as, e. g., $\mathrm{Nb}$ films [14], $\mathrm{Bi}_{2} \mathrm{Sr}_{2} \mathrm{CaCu}_{2} \mathrm{O}_{8}$ and $\mathrm{YBa}_{2} \mathrm{Cu}_{3} \mathrm{O}_{7-x}$ single crystals [15], $\mathrm{Tl}_{2} \mathrm{Ba}_{2} \mathrm{CuO}_{6+x}$ and $\mathrm{YBa}_{2} \mathrm{Cu}_{3} \mathrm{O}_{7-x}$ thin films [16], and assume $L$ as a characteristic width of flux droplets or fingers of size $\sim 10 \ldots 100 \mu \mathrm{m}[14-16]$. If such a scenario is correct, a combined effect of a non-uniform current flow due to the fractal-type flux penetration and TMI can be expected.

We note further that local heating leading to avalanche-like flux penetration in $\mathrm{Tl}_{2} \mathrm{Ba}_{2} \mathrm{CuO}_{6+x}$ and $\mathrm{YBa}_{2} \mathrm{Cu}_{3} \mathrm{O}_{7-x}$ thin films has been proposed in Ref. [16]. The dependence of the fingering on thermal conditions as well as on the sample size was also reported for untwined $\mathrm{YBa}_{2} \mathrm{Cu}_{3} \mathrm{O}_{7-x}$ single crystal [17]. Further theoretical and experimental work is needed to clarify the joint contributions of the TMI and non-uniform current flow to the development of the SMP in numerous superconductors as well as in $(\mathrm{Ba}, \mathrm{K}) \mathrm{BiO}_{3}[18]$.

To conclude, we reported the vanishing of the SMP decreasing the sample size in isotropic non-cuprate high- $T_{c}$ superconductor $\mathrm{Ba}_{0.63} \mathrm{~K}_{0.37} \mathrm{BiO}_{3}$. The results provide evidence that the SMP is not related to the enhancement of the pinning efficiency of vortices and strongly suggest that the SMP originates from a thermomagnetic instability effect and/or from a non-uniform current flow. 


\section{Acknowledgements}

The authors thank F. Mrowka and A. V. Pan for assistance. This work is partially supported by the German Israeli Foundation for Scientific Research and Development under G-553-191.14/97.

\section{References}

[1] Y. Kopelevich and P. Esquinazi, J. Low Temp. Phys. 113, 1 (1998).

[2] P. Esquinazi, A. Setzer, D. Fuchs, Y. Kopelevich, E. Zeldov, and C. Assmann, Phys. Rev. B 60, 12454 (1999).

[3] Y. Kopelevich, S. Moehlecke, J.H.S. Torres, R. Ricardo da Silva and P. Esquinazi, J. Low Temp. Phys. 116, 261 (1999).

[4] A. Gurevich and V. M. Vinokur, Phys. Rev. Lett. 83, 3037 (1999).

[5] S. V. Shiryaev, S. N. Barilo, V. I. Gatalskaya, A. V. Pushkarev, V. M. Pan., J. Cryst. Growth 172, 396 (1997).

[6] S. N. Barilo, S. V. Shiryaev, V. I. Gatalskaya, J. W. Lynn, M. Baran, H. Szymczak, R. Szymczak, and D. Dew-Hughes, Phys. Rev. B 58, 12355 (1998).

[7] For a review see E. H. Brandt, Rep. Prog. Phys. 58, 1465 (1995) and referencies therein.

[8] I. Joumard, T. Klein, P. Samuely, P. Szabo, A. G. M. Jansen, G. Fillion, H. Rakoto, J. M. Broto, and J. Marcus, Physica C 317-318, 436 (1999).

[9] D. Ertas and D. R. Nelson, Physica C 272, 79 (1996).

[10] V. Vinokur, B. Khaykovich, E. Zeldov, M. Konczykowski, R. A. Doyle, and P. H. Kes, Physica C 295, 209 (1998).

[11] P. S. Swartz and C. P. Bean, J. Appl. Phys. 39, 4991 (1968).

[12] M. Däumling and D. C. Larbalestier, Phys. Rev. B 40, 9350 (1989).

[13] C. B. Nunes, R. W. Heussner, and D. C. Larbalestier, J. Appl. Phys. 80, 1647 (1996).

[14] C. A. Durán, P. L. Gammel, R. E. Miller, and D. J. Bishop, Phys. Rev. B 52, 75 (1995).

[15] M. V. Indenbom, C. J. van der Beek, V. Berseth, M. Konczykowski, F. Holtzberg, W. Benoit, Czechoslovak Journal of Physics 46, 1451 (1996).

[16] R. Surdeanu, R. J. Wijngaarden, B. Dam, J. Rector, R. Griessen, C. Rossel, Z. F. Ren, and J. H. Wang, Phys. Rev. B 58, 12467 (1998). 
[17] M. R. Koblischka, T. H. Johansen, M. Baziljevich, H. Hauglin, H. Bratsberg, and B. Ya. Shapiro, Europhys. Lett. 41, 419 (1998).

[18] In a recently published letter I. Joumard et al. (Phys. Rev. Lett. 82, 4930 (1999)) reported the decrease of the neutron diffracted intensity as the applied field increased to values close to the onset of the SMP in a $(\mathrm{Ba}, \mathrm{K}) \mathrm{BiO}_{3}$ superconducting sample. The authors suggested that the SMP is related to a change in the vortex structure of the vortex solid. However, the non-observation of a diffracted intensity may be related to a low experimental resolution rather than to a real change in vortex structure. In fact, higher resolution experiments using second order neutron diffraction measurements reveal a well ordered FLL at fields above the $\mathrm{SMP}$ in $\mathrm{Bi}_{2} \mathrm{Sr}_{2} \mathrm{CaCu}_{2} \mathrm{O}_{8}$ high- $T_{c}$ superconductor, see E. M. Forgan et al., Czechoslovak Journal of Physics 46, 1571 (1996). 